\title{
Моделирование тепловых режимов подъемного участка дефлегматора бытового абсорбционного холодильного агрегата
}

\author{
О. Б. Васылив, А. С. Титлов ${ }^{\bowtie}$, А. О. Холодков \\ Одесская национальная академия пищевых технологий, Канатная, 112, Одесса, 65039 \\ $\bowtie$ e-mail: titlov1959@gmail.com
}

\begin{abstract}
Выполнено математическое моделирование тепловых режимов дефлегматора, которые отвечают за процессы очистки и транспортировке пара аммиака. Моделирование проведено на типовых конструкциях абсорбционных холодильных агрегатов с учетом обоснованных допущений и результатов собственных экспериментальных исследований. В основе модельных представлений лежат законы сохранения тепла и массы. В результате была разработана методика расчета тепловых режимов работы дерлегматора, применимая для типовых конструкций бытовых абсорбционных холодильных агрегатов.
\end{abstract}

Ключевые слова: тепловые режимы; энергетическая эффективность; абсорбционный холодильник; дефлегматор; тепловая изоляция.

\section{Моделювання теплових режимів під'ємної ділянки дефлегматора побутового абсорбційного холодильного агрегату}

\author{
О. Б. Василів, О. С. Титлов, А. О. Холодков \\ Одеська національна академія харчових технологій, Канатна, 112, Одеса, 65039
}

\begin{abstract}
Виконано математичне моделювання теплових режимів дефлегматора, які відповідають за процеси очищення та транспортування пара аміаку. Моделювання проведено на типових конструкціях абсорбційних холодильних агрегатов з урахуванням обгрунтованих припущень і результатів власних експериментальних досліджень. В основі модельних уявлень лежать закони збереження тепла і маси. В результаті була розроблена методика розрахунку теплових режимів роботи дефлегматора, що може бути застосована для типових конструкцій побутових абсорбційних холодильних агрегатів.
\end{abstract}

Ключові слова: теплові режими; енергетична ефективність; абсорбційний холодильник; дефлегматор; теплова ізоляція.

DOI: $\underline{\text { http: }: / / d x . d o i . o r g / 10.15673 / r e t . v 53 i 1.535 ~}$

(C) The Author(s) 2017. This article is an open access publication

This work is licensed under the Creative Commons Attribution 4.0 International License (CC BY) http://creativecommons.org/licenses/by/4.0/

\section{Введение}

Специфика работы абсорбционных аппаратов предопределяет их высокую тепловую инерционность, т.е. длительный пусковой период.

Основной вклад в тепловую инерционность абсорбционного аппарата вносит холодильная (морозильная) камера. Это связано с ограниченной холодопроизводительностью АХА, а, следовательно, с необходимостью установки дополнительной, по сравнению с компрессионными моделями, теплоизоляции. Такое положение наиболее характерно для морозильных камер и двухкамерных моделей, полезный объем которых обычно не менее 180 дм³ $^{3}$ а толщина теплоизоляцион- ных покрытий, выполненных из пенополиуретана, составляет 0,10...0,12 м [1]. Несмотря на то, что время выхода на рабочий режим таких аппаратов из «отепленного» состояния достаточно велико (10...18 часов), высокая тепловая инерционность камер позволяет уменьшить воздействие окружающей среды в режиме циклической работы АХА (КРВ <1), по сравнению с компрессионными моделями. Вместе с тем, как было отмечено ранее, существующие методики $[2,3]$ позволяют производить расчет теплопритоков в камеру холодильных аппаратов, т.е. определить необходимую величину холодопроизводительности испарителя АХА, только в стационарных условиях и не могут быть использованы для прогнозирования температурных ре- 
жимов камер при работе с КРВ < 1, которая обусловлена функционированием системы регулирования при температурах окружающей среды ниже $32^{\circ} \mathrm{C}$ (либо $\left.43^{\circ} \mathrm{C}\right)[4]$.

Аналогично состояние вопроса и для AXA, работающей в режиме циклической тепловой нагрузки на термосифон.

Вместе с тем для случая АХА необходимо отметить следующее. Так как абсорбционные аппараты проектируются изначально на "жесткие" условия эксплуатации, то при снижении температуры окружающей среды, а, следовательно, и теплопритоков в камеру, все основные элементы AXА, за исключением дефлегматора, имеют возможность работы в более благоприятных условиях, т.е. с «запасом».

Дефлегматор, который также спроектирован для работы в «жестких» условиях, в этом случае, дополнительно к своей прямой функции - очистки паров аммиака от паров воды, задерживает и часть аммиака, поступающего в конденсатор. При этом, очевидно, что чем ниже температура окружающей среды, тем большей будет эта часть.

В конечном счете, снижение температуры окружающей среды приводит к снижению холодопроизводительности при неизменной тепловой нагрузке на термосифон, т.е. снижению КПД холодильного аппарата.

Остается также неизученным вопрос о применимости в реальных системах (КРВ < 1), зависимостей найденных для стационарных условий [5], связывающих оптимальную работу испарителя АХA и температурный скачок в характерной точке дефлегматора.

Таким образом, задачей данного исследования является разработка метода математического моделирования дефлегматора AXA.

\section{1 Исходные предпосылки}

Режимы работы дефлегматора в значительной мере, определяют эффективность работы АХА. При этом следует отметить что, несмотря на то, что функциональным предназначением дефлегматора является очистка паров аммиака от паров воды с отводом флегмы в ректификатор, практически всегда имеется зона, в которой происходит частичная конденсация чистых паров аммиака. Очевидно, что в этом случае снижается и холодопроизводительность испарителя, и тепловой коэффициент АХА. Необходимо также отметить, что за исключением конструкций АХА с трехпоточным генераторным узлом [6], в которых в дефлегматор поступает практически чистый пар аммиака и он полностью закрыт теплоизоляцией, в подавляющем большинстве современных конструкций [1] дефлегматор теплоизо- лирован лишь частично, а так как он, как и все остальные элементы AXA, проектируется для работы в "жестких" условиях $\left(t_{\text {o.c. }}=32^{\circ} \mathrm{C}\right)-$ при снижении температуры окружающей среды возрастает и количество непоступившего в испаритель хладагента.

При снижении $t_{\text {o.c. }}$ реальные АХА, несмотря на некоторое снижение холодопроизводительности, обеспечивают требуемые температурные режимы в холодильных камерах, так как одновременно происходит и снижение теплопритоков из окружающей среды. Очевидно, что в этом случае энергетическая эффективность AХА ниже, чем при работе в «жестких» условиях.

С другой стороны, при снижении $t_{\text {o.c. }}$ и работе дефлегматора по своему функциональному назначению появляется возможность уменьшить количество генерируемого пара хладагента, т.е. уменьшить величину $Q_{T}$, а в конечном счете повысить энергетическую эффективность абсорбционного холодильного аппарата.

Следует также иметь в виду, что для реализации таких энергосберегающих режимов весь дефлегматор необходимо закрыть тепловой изоляцией, что позволит снизить количество непоступившего аммиака в испаритель при снижении $t_{\text {o.c. }}$ и $Q_{T}$.

Для нахождения оптимальных режимов работы дефлегматора в широком диапазоне изменения $t_{\text {o.c. }}$ необходимо разработать его математическую модель.

С помощью математической модели при заданных геометрических и теплотехнических параметрах дефлегматора ( $\left.L_{D}, d_{B H}, d_{H}, \lambda_{c m}\right)$ и теплоизоляционного кожуха $\left(d_{u 3}, \lambda_{u 3}\right)$, а также параметрах парового потока на входе (температура $\vartheta_{b x}$, концентрация $\xi_{6 x}^{\prime \prime}$ и массовый расход $G_{b x}$ ), которые определяются величиной $Q_{T}$, можно определить и параметры парового потока на выходе дефлегматора $\left(\vartheta_{\text {вblx }}, \xi_{b b l x}^{\prime \prime}, G_{b b x}\right)$ при различHых $t_{\text {o.c. }}$.

При выполнении дефлегматором своих функций $\vartheta_{\text {вых }}$ должна соответствовать температуре насыщения аммиака $\left(\vartheta_{\mathrm{NH}_{3}}^{S}\right)$, а $\xi_{\text {выx }}^{\prime \prime}=1$.

Зная $G_{b b l x}=f_{1}\left(Q_{T}\right)$, а $Q_{0}=f_{2}\left(G_{\text {выx }}\right)$, можно найти зависимость $Q_{0}=f_{3}\left(Q_{T}\right)$ для данной конструкции дефлегматора и генераторного узла АХA.

\section{2 Математическая модель}

Функциональная схема подъемного участка дефлегматора AXА представлена на рисунке 1.

На вход дефлегматора поступает паровой поток с параметрами $\vartheta_{b x}, \xi_{b x}^{\prime \prime}, G_{B x}$. 


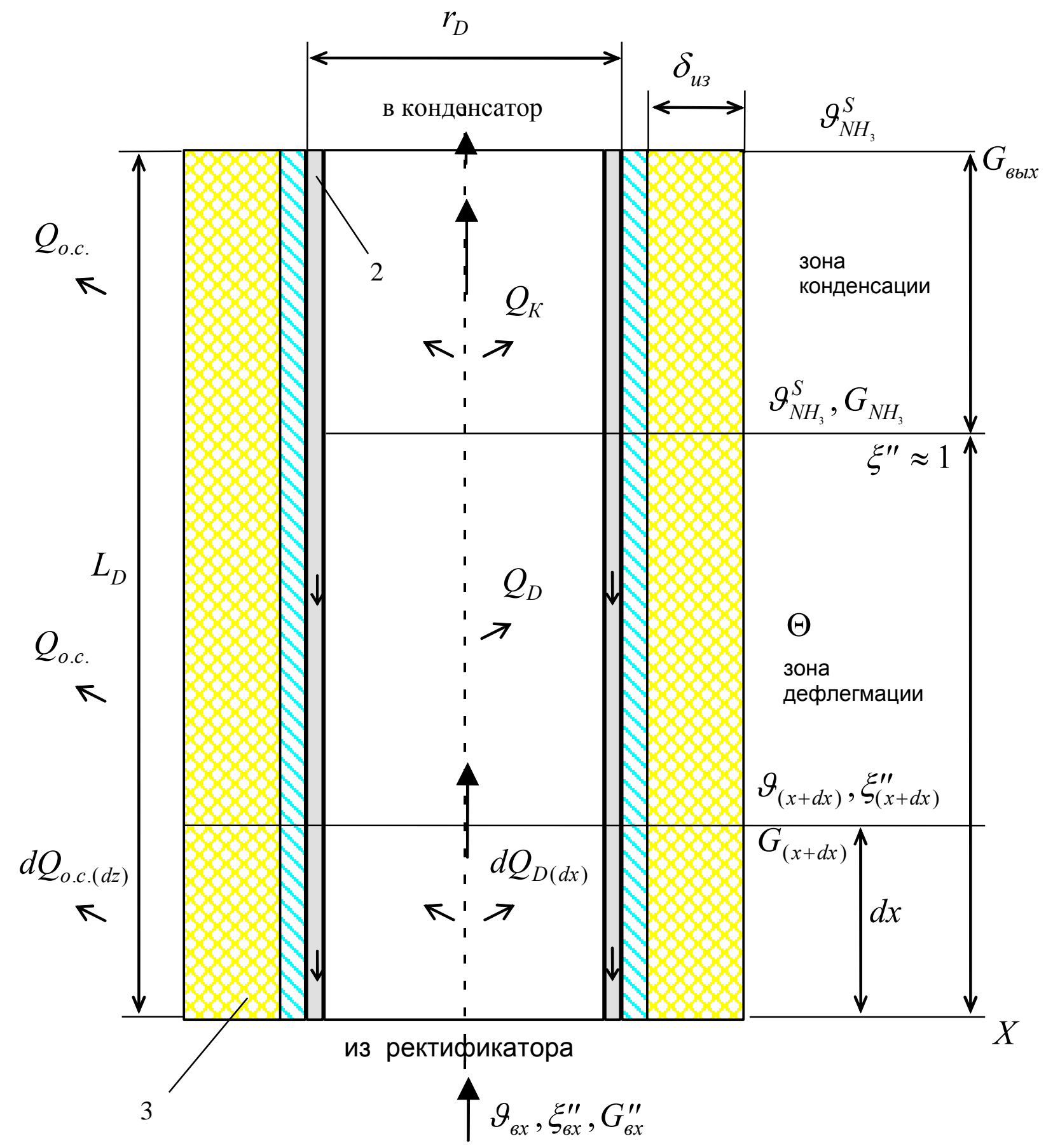

1 - стекающая флегма; 2 - стенка дефлегматора; 3 - теплоизоляционный кожух дефлегматора

Рисунок 1 - Схема работы дефлегматора АXА 
Противотоком ему движется стекающая флегма с параметрами $T, \xi^{\prime}, G_{\phi}$. Теплота дефлегмации, после подогрева флегмы, отводится через стенку и теплоизоляционное покрытие в окружающую среду.

В общем случае, исходя из физических представлений [56], можно выделить два режима работы дефлегматора:

а) режим «очистки» паровой смеси, когда температура стенки превышает температуру конденсации аммиака при рабочем давлении;

б) режим частичной конденсации чистых паров аммиака $\left(t_{c m} \approx \vartheta_{\mathrm{NH}_{3}}^{S}\right)$.

При моделировании использовались следующие допущения:

а) термическое сопротивление и теплоемкость пленки флегмы незначительны и при моделировании тепловых режимов дефлегматора ими можно пренебречь;

б) температуры флегмы и стенки дефлегматора равны и на элементарном участке $d x$ постоянны.

В основе математической модели лежат уравнения сохранения тепла и массы, которые для элементарного участка $d x$ имеют вид:

$$
\begin{gathered}
d Q_{D(d x)}=d Q_{\text {o.c. }(d x)} \\
G_{(x)}^{\prime \prime}=G_{(x+d x)}^{\prime \prime}+G_{(x)}^{\prime}
\end{gathered}
$$

Уравнение (1) можно записать в виде:

$$
\begin{aligned}
& d\left(G^{\prime \prime} i\right)_{(d x)}=d\left[G_{(x)}^{\prime \prime} i\left(\vartheta_{(x)} \xi_{(x)}^{\prime \prime}\right)-G_{(x+d x)}^{\prime \prime} i\left(\vartheta_{(x+d x)} \xi_{(x+d x)}^{\prime \prime}\right)\right]= \\
& \alpha(\vartheta-T) d F_{1}+k\left(T-t_{\text {o.c. }}\right) d F_{2}
\end{aligned}
$$

где $\alpha$ - коэффициент теплоотдачи от парового потока к стекающей флегме, определяемый на основе известных соотношений [7];

$\vartheta, T$ - температуры на участке $d x$ парового потока и стенки (флегмы), соответственно;

$\boldsymbol{k}$ - коэффициент теплопередачи между стекающей флегмой и окружающей средой;

$d F_{1}$ и $d F_{2}$ - площади элементарного участка $d x$ в процессах теплообмена.

$$
\begin{aligned}
& \text { Обозначим } A=\alpha \pi d_{\text {вн }} \text { и } \\
& B=\frac{\pi}{\frac{1}{\alpha_{\text {o.c. }} d_{u 3}}+\frac{2}{\lambda_{c m}} \ln \frac{d_{H}}{d_{\text {BH }}}+\frac{2}{\lambda_{u 3}} \ln \frac{d_{u 3}}{d_{H}}} .
\end{aligned}
$$

Тогда уравнение (1) будет иметь вид:

$$
d\left(G^{\prime \prime} i\right)_{(d x)}=A(\vartheta-T) d x+B\left(T-t_{\text {o.c. }}\right) d x
$$

Для нахождения неизвестных параметров парового потока на выходе участка $d x$ к уравнениям (1) и (2) добавляются:

$$
\begin{gathered}
\xi_{(x)}^{\prime}=\frac{G_{X(x)}^{\prime}}{G_{X(x)}^{\prime}+G_{A(x)}^{\prime}} \\
\xi_{(x+d x)}^{\prime \prime}=\frac{G_{X(x)}^{\prime \prime}-G_{X(x)}^{\prime}}{G_{X(x)}^{\prime \prime}-G_{X(x)}^{\prime}+G_{A(x)}^{\prime \prime}-G_{A(x)}^{\prime}}, \\
\xi_{(x)}^{\prime \prime}=\frac{G_{X(x)}^{\prime \prime}}{G_{X(x)}^{\prime \prime}+G_{A(x)}^{\prime \prime}}, \\
\xi_{(x)}^{\prime}=f_{4}(T, P), \\
G_{(x)}^{\prime}=G_{X(x)}^{\prime}+G_{A(x)}^{\prime} .
\end{gathered}
$$

Решить систему уравнений можно следующим образом.

В первом приближении принимаем на участке $d x-\vartheta=$ const и равной входным параметрам парового потока, т.е. $\vartheta=\vartheta_{\text {вx }}$.

Так как теплоте дефлегмации фактически соответствует теплота фазового перехода, то левую часть уравнения (3) можно записать как:

$$
d\left(G^{\prime \prime} i\right)_{(d x)}=G_{X}^{\prime} r
$$

где $r$ - теплота парообразования паровой смеси при $\vartheta_{6 x}$ и $P$.

Тогда из можно записать зависимость для температуры стенки (флегмы) на участке $d x$ :

$$
T=\frac{A \vartheta-\frac{G_{X}^{\prime} r}{d x}-B t_{o . c .}}{A-B} .
$$

Зная зависимость характеристик генераторного узла

$$
G_{B x}^{\prime \prime}=G_{X(x)}^{\prime \prime}+G_{A(x)}^{\prime \prime}=f_{5}\left(Q_{T}\right)
$$

n

$$
\xi_{8 x}^{\prime \prime}=\xi_{(x)}^{\prime \prime}=f_{6}\left(Q_{T}\right)
$$

для входного сечения, можно найти зависимости:

$$
\begin{gathered}
G_{X(x)}^{\prime \prime}=f_{5}\left(Q_{T}\right) \cdot f_{6}\left(Q_{T}\right), \\
G_{A(x)}^{\prime \prime}=f_{5}\left(Q_{T}\right) \cdot\left[1-f_{6}\left(Q_{T}\right)\right] .
\end{gathered}
$$

С учетом вышеприведенных соотношений

$$
G_{X(x+d x)}^{\prime \prime}=G_{X(x)}^{\prime \prime}-G_{X(x)}^{\prime}-G_{A(x)}^{\prime}
$$

далее решаем систему из трех уравнений, в результате чего определяем неизвестные параметры флегмы на участке $d x\left(G_{X(x)}^{\prime}\right.$ и $\left.G_{A(x)}^{\prime}\right)$ и парового потока на выходе участка $d x\left(\xi_{(x+d x)}^{\prime \prime}\right.$ и $\left.\vartheta_{(x+d x)}=f_{7}\left(\xi_{(x+d x)}, P\right)\right)$.

На последующих этапах производится корректировка текущих параметров с принятием 


$$
\vartheta=\frac{\vartheta_{6 x}+\vartheta_{(x+d x)}}{2}
$$

до достижения заданной точности.

В дальнейшем по приведенному алгоритму производится расчет каждого элементарного участка $d x$ дефлегматора до достижения $\xi^{\prime \prime} \approx 1$, а $\vartheta \approx \vartheta_{N H_{3}}^{S}$.

При этих параметрах дефлегматор начинает выполнять функции конденсатора и расчет проводится по известным методикам [8-10] с фиксацией количества сжиженного аммиака и поступившего в конденсатор.

В первом приближении диаметр теплоизоляции, при заданных $L_{D}, d_{H} n d_{в н}$, может быть найден из условия обеспечения холодопроизводительности $Q=f_{2}\left(G_{\text {выx }}\right)$ в «жестком» режиме работы холодильного аппарата $\left(t_{\text {o.c. }}=32^{\circ} \mathrm{C}\right)$ :

$$
G_{b x} i\left(\vartheta_{b x}, \xi_{b x}^{\prime \prime}\right)-G_{6 b l x} i\left(\vartheta_{N H_{3}}^{S}, \xi_{b b l x}^{\prime \prime}=1\right)=\frac{\left(\bar{\vartheta}-t_{o . c .}\right)}{R_{D}}
$$

где $\bar{\vartheta}$ - средняя температура насыщения парового потока, определяемая как: $\bar{\vartheta}=\frac{\vartheta_{6 x}+\vartheta_{N H_{3}}^{S}}{2}$;

$R_{D}$ - термическое сопротивление дефлегматора, учитывающее и теплоотдачу парового потока к стенке.

В случае, когда при принятом в первом приближении диаметре теплоизоляции дефлегматор не справляется со своими функциями $\left(\xi_{\text {sыx }}^{\prime \prime}<1\right)$, необходимо идти на его уменьшение до достижения $\xi_{\text {сыx }} \approx 1$.

Таким образом, задаваясь геометрическими параметрами дефлегматора и теплоизоляционного кожуха, можно определить параметры парового потока $\vartheta_{\text {gblx }}, G_{\text {gbx }}$ и $\xi_{\text {gblx }}^{\prime \prime}$ при различных величинах $Q_{T}$ и $t_{\text {o.c. }}$.

При этом очевидным является и то, что максимальная холодопроизводительность АХА достигается при $\xi_{\text {выx }}^{\prime \prime} \approx 1$ на выходе дефлегматора. Поэтому для каждой конструкции необходимо найти зависимости

$$
Q_{T}=f_{8}\left(Q_{0(\max )}, t_{\text {o.c. }}=\mathrm{var}\right),
$$

удовлетворяющие этому условию.

Такая зависимость может быть эффективно использована в аппаратах, работающих в циклическом режиме.

Для аппаратов, работающих в режиме постоянной $Q_{T}$ с изменением ее абсолютной величины в зависимости от требуемой холодопроизводительности при различных $t_{\text {o.c. }}$, может быть получена зависимость:

$$
Q_{T}=f_{9}\left(Q_{0(i)}, t_{\text {o.c. }}=\mathrm{var}\right),
$$

Таким образом, разработанная методика позволит найти оптимальные соотношения между рабочими характеристиками генераторного узла и испарителем в широком диапазоне температур окружающей среды, которые могут быть использованы в системах регулирования холодильных аппаратов абсорбционного типа.

Для практической реализации методики необходимо провести экспериментальные исследования серийных и опытных образцов аппаратов с AXА, которые позволят оценить правомерность принятых допущений и эффективность используемых при моделировании соотношений, а также определить граничные условия при различных режимах работы генераторного узла.

\section{Выводы}

1. АХП обладая рядом несомненных эксплуатационных преимуществ (надежность, длительный ресурс, бесшумность в работе, минимальная стоимость) перед компрессионными аналогами, а также универсальностью в использовании источников энергии, при наличии энергосберегающих технологий могут расширить свое присутствие на рынке бытовой холодильной техники.

2. Одним из эффективных и малобюджетных методов повышения энергетической эффективности АХП является технология снижения потерь при транспортировке аммиака в зону производства искусственного холода (испаритель). Ключевую роль в этом процессе выполняет дефлегматор АХА, который очищает пар аммиака за счет отвода тепла фазового перехода в окружающую среду в диапазоне температур от 10 до $32^{\circ} \mathrm{C}$.

3. Моделирование тепловых режимов дефлегматора, выполненное в рамках настоящей работы, позволяет получить оптимальные параметры тепловой изоляции.

4. Особое значение данное исследование имеет для систем энергосберегающего управления AXА, которые используют индикаторы температур в характерных точках дефлегматора для выработки управляющего воздействия.

\section{Литература}

1. Бабакин, Б. С. Бытовые холодильники и морозильники / Б. С. Бабакин, В. А. Выгодин. - Рязань : Узоречье, 2005. -860 c.

2. Морозюк, л. И. Исследование генераторов АДХМ / Л.И. Морозюк, Н.Ф. Хоменко, А.Н. Галавацкий //Холод. техн. и технолог. Киев. - 1981. - № 12. - С. 21-25.

3. Янченко, В. М. Математическое моделирование теплопотерь в горячих узлах АДХМ // Машины и аппараты холод., криогенной техники и кондиционирования воздуха / В.М. Янченко, А.В. Котельников. - Л., 1976. - № 1. - С. 79-83.

4. ГОСТ 16317-95 (ИСО 5155-83, иСО 7371-85, МЭК 335-2-24-84). Приборы холодильные электрические бытовые. Общие технические условия. - М.: Изд-во стандартов, 1995. ДСТУ 2295-93. 
5. Титлов, А. С. Оптимизация температурно-энергетических характеристик абсорбционно-диффузионных холодильных агрегатов и аппаратов бытовой техники на их основе / А.С. Титлов, Ю.С. Ботук, А.В. Мазур, В.В. Завертаный // Тепловые режимы и охлаждение радиоэлектронной аппаратуры. - 1995. - № 1-2. - С. 69-78.

6. Тітлов, О. С. Сучасні тенденці розвитку побутової абсорбційної холодильної техніки / О.С. Тітлов // Наукові праці Одеської державної академії харчових технологій. - 1998. - № 18. - С. 205-208.

7. Справочник по теплообменникам: в 2-х т. Т. 1. - М. : Энергоатомиздат, 1987. - 560 с.

8. Морозюк, Л. И. Системный анализ генераторов абсорбционно-диффузионных термотрансформаторов / л. И. Морозюк // Холодильная техника и технология. 1999. - № 61. - С. 36-41.

9. Морозюк, Л. И. Основы функционального проектирования термосифонных насосов абсорбционнодиффузионных холодильных машин (часть 1) / Л. И. Морозюк, А. В. Пащенко // Холодильная техника и технология. -2000 . - № 65. - С. 66-71.

10. Морозюк, Л. И. Определение конструктивных характеристик термосифонных насосов абсорбционнодиффузионных холодильных машин (Часть 2) / Л. И. Морозюк, А. В. Пащенко // Холодильная техника и технология. - 2000. - № 66. - С. 51-55.

Отримана в редакції 14.12.2016, прийнята до друку 07.02.2017

\title{
Thermal Modes Simulation of Household Absorption-Type Refrigerating Unit Rectifier Lift Site
}

\author{
O. B. Vasyliv, A. S. Titlov ${ }^{\bowtie}$, A. O. Kholodkov
}

Odessa National Academy of Food Technologies, 112 Kanatnaya st.,Odessa, 65039, Ukraine

$\triangle$ e-mail: titlov1959@gmail.com

\begin{abstract}
Currently, developers of modern refrigeration equipment, in accordance with the plans of the UN, are moving to natural refrigerants (hydrocarbons, carbon dioxide and ammonia) that do not have an adverse technological impact on the ecosystem of the planet. In domestic refrigeration technology, one of the options is absorption refrigerator, the working body of which is water-ammonia mixture with addition of hydrogen. Having a number of unique advantages over compression analogs, absorption systems are characterized by lower energy characteristics. Since the absorption apparatus is designed initially to "hard" operating conditions, then with a decrease in the ambient temperature, and, consequently, heat flows into the chamber, all the basic elements of the ARU, with the exception of the reflux condenser, are able to operate under more favorable conditions. A reflux condenser, which is also designed to operate under "hard" conditions, in this case, in addition to its direct function of cleaning ammonia vapors from water vapor, also detains part of the ammonia entering the condenser. At the same time, it is obvious that the lower the ambient temperature, the greater this part will be. Ultimately, a decrease in the ambient temperature results in a decrease in the cooling capacity with a constant thermal load on the thermosyphon, COP of the refrigeration unit. As the analysis showed, the maximum thermodynamic losses in absorption refrigeration aggregates are concentrated in the generator unit during evaporation of ammonia, its purification from water vapor and transport to the evaporator. In this connection, the mathematical modeling of the thermal modes of the reflux condenser was performed, which is responsible for the purification and transportation of ammonia vapor. Modeling was carried out on standard designs of absorption cooling units taking into account reasonable assumptions and results of own experimental researches. At the heart of model representations lie the laws of conservation of heat and mass. Due to the high thermal inertia of the processes in the rectifier, stationary operating modes were modeled. In the simulation, the flow mode of the vapor mixture in the rectifier was considered turbulent, and the radial diffusion processes were neglected. As a result, the method for calculating the thermal modes of a reflux condenser, which is applicable for typical design of household absorption refrigeration unit has been developed.
\end{abstract}

Keywords: Thermal modes; Energy efficiency; Absorption refrigerator; Rectifier; Thermal insulation.

\section{References}

1. Babakin, B. S., Vygodin, V. A. (2005) Bytovye kholodilniki i morozilniki. Ryazan, Uzorechie, 860 p. (in Russian)

2. Morozyuk, L. I. Khomenko N. F., Galavatskiy A. N. (1981) Issledovanie generatorov ADKHM. Refrigeration
Engineering and Technology, No. 12, 21-25. (in Russian) 3. Yanchenko, V. M., Kotelnikov, A. V. (1976) Matematicheskoie modelirovaniie teplopoter $\mathrm{v}$ goriachikh uzlakh ADKHM. Mashiny i apparaty kholod., kriogennoy tekhniki i konditsionirovaniya vozdukha, No. 1, 79-83. (in Russian) 
4. GOST 16317-95 (ISO 5155-83, ISO 7371-85, MEK 335-224-84). Pribory kholodilnyye elektricheskiye bytovyye. Obshchiye tekhnicheskiye usloviya. - M.: Izd-vo standartov, 1995. DSTU 2295-93. (in Russian)

5. Titlov, A. S., Botuk, Yu. S., Mazur, A. V., Zavertanyy, V. V. (1995) Optimizatsiya temperaturnoenergeticheskikh kharakteristik absorbtsionno-diffuzionnykh kholodil'nykh agregatov i apparatov bytovoy tekhniki na ikh osnove. Teplovyie rezhimy i okhlazhdeniie radioelektronnoi apparatury, No. 1-2, 69-78. (in Russian)

6. Títlov, O. S. (1998) Suchasni tendentsii rozvytku pobutovoi absorbtsiinoi kholodyl'noi tekhniky. Research works, No. 18, 205-208. (in Ukrainian)

7. Spravochnik po teploobmennikam. Vol. 1. Moskow, Energoatomizdat, 1987, 560 p. (in Russian)
8. Morosuk, L. I. (1999) Sistemnyi analiz generatorov absorbtsionno-diffuzionnykh termotransformatorov. Refrigeration Engineering and Technology, No.61, 36-41 (in Russian)

9. Morosuk, L. I., Pashchenko, A. V. (2000) Osnovy funktsionalnogo proyektirovaniia termosifonnykh nasosov absorbtsionno-diffuzionnykh kholodilnykh mashin (chast 1). Refrigeration Engineering and Technology, No. 65, 66-71 (in Russian)

10. Morosuk, L. I., Pashchenko, A. V. (2000) Opredeleniie konstruktivnykh kharakteristik termosifonnykh nasosov absorbtsionno-diffuzionnykh kholodilnykh mashin (Chast 2) Refrigeration Engineering and Technology, No. 66, 51-55 (in Russian) 